\title{
Designing emergency response networks for hazardous materials transportation
}

\author{
Oded Berman ${ }^{\mathrm{a}}$, Vedat Verter ${ }^{\mathrm{b}, *}$, Bahar Y. Kara ${ }^{\mathrm{c}}$ \\ ${ }^{a}$ Joseph L. Rotman School of Management, University of Toronto, Canada \\ ${ }^{\mathrm{b}}$ Faculty of Management, McGill University, Montreal, Que., Canada H3A 1G5 \\ ${ }^{\mathrm{c}}$ Department of Industrial Engineering, Bilkent University, Turkey
}

Available online 10 August 2005

\begin{abstract}
Undesirable consequences of dangerous goods incidents can be mitigated by quick arrival of specialized response teams at the accident site. We present a novel methodology to determine the optimal design of a specialized team network so as to maximize its ability to respond to such incidents in a region. We show that this problem can be represented via a maximal arc-covering model. We discuss two formulations for the maximal arc-covering problem, a known one and a new one. Through computational experiments, we establish that the known formulation has excessive computational requirements for large-scale problems, whereas the alternative model constitutes a basis for an efficient heuristic. The methodology is applied to assess the emergency response capability to transport incidents, that involve gasoline, in Quebec and Ontario. We point out the possibility of a significant improvement via relocation of the existing specialized teams, which are currently stationed at the shipment origins.
\end{abstract}

(c) 2005 Elsevier Ltd. All rights reserved.

Keywords: Hazardous materials; Transportation; Emergency response; Maximal cover; Geographical information systems

\section{Introduction}

A substantial amount of hazardous materials (hazmats), such as flammable liquids and poisonous gases, need to be transported prior to their consumption or disposal. The US Department of Transportation [1],

\footnotetext{
* Corresponding author. Fax: +1 5143983876.

E-mail addresses: berman@rotman.utoronto.ca (O. Berman), vedat.verter@mcgill.ca (V. Verter), bkara@bilkent.edu.tr (B.Y. Kara).
}

0305-0548/\$ - see front matter (C) 2005 Elsevier Ltd. All rights reserved. doi:10.1016/j.cor.2005.06.006 
for example, estimates that there were 300 million dangerous goods shipments in 1998 and forecasts a $2 \%$ annual growth in the amount of hazmats produced in the country. Despite widespread deregulation of transport industry, mitigation of the public and environmental risks associated with these shipments remains a government responsibility. A number of transport safety measures aim at reducing the likelihood and possible undesirable consequences of incidents involving accidental release of dangerous goods during transportation. This proactive group of policy tools includes the use of low-risk routes for hazmat shipments, regulatory requirements for driver training, driving hours and container specifications, inspections to enforce compliance, and closure of certain road segments to vehicles carrying dangerous goods. Nonetheless, accidents do happen. During the 1990-1998 period, for example, there were an average of 410 serious hazmat incidents in the US annually, which caused fatalities, injuries, evacuation of homes and businesses as well as property damage [1]. When a hazmat incident occurs, effective emergency response is crucial for containing its impact to the smallest possible area around the accident site. Clearly, such reactive safety measures complement the proactive policies in mitigating undesirable consequences of transport incidents. The establishment of a hazmat emergency response system, which is the focus of this paper, constitutes a building block toward a comprehensive public and environmental safety infrastructure.

The firefighters, paramedics and police in the vicinity of accident site are typically first to respond to a transport accident. It is important that they quickly identify the type of hazmat involved (if any) in the incident and take precautions to protect the general public during the initial response phase. To facilitate this, the US, Canada and Mexico require each potentially hazardous vehicle to carry a placard indicating the type (4-digit ID number) of its cargo. Also, a guidebook is developed jointly by the three transport ministries to aid the first-responders e.g., the area to be evacuated depending on hazmat type and the size of spill [2]. In most cases, however, it is necessary to send a specialized hazmat response team and equipment to the accident site. It is crucial that the availability and involvement of such specialized teams are planned prior to dangerous goods shipments. In Canada, the 1992 Transportation of Dangerous Goods Act, in fact, requires each shipper to have an approved emergency response assistance plan (ERAP) before transporting or importing dangerous goods. An ERAP must provide a description of the arrangements for specialized emergency response, a potential accident assessment as well as a contact person the firstresponders need to call to activate the plan. The final phase of emergency response to hazmat incidents is the cleanup of the accident site, which is usually overseen by an environmental protection agency with jurisdiction.

The current practice and regulation of dangerous goods emergency response emphasize the availability of information, the coordination among responders and the procedures that need to be followed at the accident site. Although accidents can occur during loading and unloading of hazmats, our focus in this paper is on possible incidents during transportation. The time it takes for the specialized response team to arrive at the accident site is another crucial factor, which seems to be overlooked by regulators. In Canada, for example, there are no established response time standards that are used by Transport Canada in the approval process of ERAPs [3]. The importance of quick response in alleviating undesirable consequences of hazmat incidents, however, has been recognized in a number of occasions. One example is the 1998 derailment of a CSX freight train in West Virginia that resulted in the release of 21,500 gallons of formaldehyde solution from two railcars. In a safety recommendation report following this accident, US National Transportation Safety Board [4] stated that the three-hour delay in the arrival of specialized teams could have had serious consequences had the leaking chemicals been more hazardous than formaldehyde. A recent report by the same agency recommended the inclusion of railroad mileposts 
in emergency response maps throughout the US so as to minimize response times [5]. In January 2002, the governor of North Dakota highlighted the fast response of emergency management teams to a Canadian Pacific Railway train derailment as the main factor that kept the damage caused by this spectacular accident $^{1}$ relatively low [6].

In this paper, we provide a methodology for determining the optimal locations of specialized response team stations so as to maximize their ability to respond to hazmat incidents within a threshold time. We assume that the number of stations to be established is pre-determined. The required equipment, expertise and response time varies with the characteristics of dangerous good involved in the incident. Therefore, a separate emergency response station network needs to be established for each hazmat category that is shipped in high volumes across a region. The concept of location coverage is pertinent for representing the objective of our problem. If a potential incident site can be reached from one of the emergency response stations within the threshold time, then this location is considered covered. Note that the incident can occur anywhere on the transport network used for dangerous goods shipments. The number of specialized teams is often insufficient to cover the entire transport network and the road links with higher risk need to be prioritized. In a critical overview, Erkut and Verter [7] identify population exposure, incident probability and expected risk (i.e., probability times exposure) as the prevailing models for hazmat transport risk. These risk attributes determine the relative significance of providing emergency response coverage to each road link.

The hazmat emergency response network design problem can be solved via a maximal arc-covering location model. This is an extension of the maximal covering location model, which has been used in siting non-hazmat emergency services such as fire stations, ambulance stations and blood banks (see [8] for references). The original model, developed by Church and ReVelle [9], seeks to maximize the coverage provided to a set of demand points each corresponding to a population center. Point coverage has remained as a common structural property of the numerous extensions proposed within the past three decades. We refer the reader to Marianov and Serra [10] for a recent review of this literature. The distinguishing feature of hazmat emergency response is that it requires coverage of a set of arcs that make up the transport network rather than a set of points. There has been limited work on the maximal arccovering location problem. It was first introduced within the context of set covering models as described in [11] and later extended to the maximal covering location model in [12]. They proved that an optimal solution to the problem exists in a finite dominating set of points which they call NIPS. Using NIPS they formulated the problem as an integer linear program similar to that of the maximal (node) covering location problem (MCLP). In this paper, we also present an alternative formulation of the problem. We are unaware of any other previous work on the maximal arc-covering location model.

The remainder of the paper is organized as follows. The problem is defined in Section 2 where we also discuss two formulations and methods to solve the problem. Section 3 reports on our computational experiments that establish the need for the development of a heuristic procedure as well as the efficiency of a greedy heuristic based on our formulation. An application of our methodology for designing an emergency response system to gasoline incidents in Quebec and Ontario highways is presented in Section 4. Section 5 provides our concluding comments.

\footnotetext{
1 240,000 gallons of anhydrous ammonia were released from eight ruptured tank-cars.
} 


\section{The problem}

Let $G=(N, A)$ be a network with a nodal set $N$ and an $\operatorname{arc} \operatorname{set} A$. Associated with each $\operatorname{arc}(i, j)$ there is a weight $w_{i j}$ which represents the benefit of covering entirely arc $(i, j) \in A$. In the context of hazmat transportation, the arcs correspond to road links whereas the nodes represent shipment origins/destinations, potential response facility locations and road intersections. In this paper, we use population exposure as the measure of transport risk, and hence arc weights are the number of people within a threshold distance of the road link multiplied by the number of hazmat trucks shipped across that link. Let $d_{i j}$ designate the length of arc $(i, j)$ and $d(x, y)$ the shortest distance between any pair of points $x, y \in G$. We denote by $p$ the number of (response) facilities to be located on the network.

The maximal arc-covering problem involves locating $p$ facilities so as to maximize the total weighted arc length covered. A point $y \in G$ is covered by a facility at $x \in G$ if $d(x, y) \leqslant R$, where $R$ is the pre-determined coverage distance. Let $Z_{k l}(S)$ denote the fraction of arc $(k, l) \in A$ covered by a set of facilities located at $S \subseteq G$ (i.e., $0 \leqslant Z_{k l}(S) \leqslant 1$ ). Thus, the problem is

$$
\max _{\substack{S \subseteq G \\|S|=p}} w(S)=\sum_{(k, l) \in A} w_{k l} Z_{k l}(S) .
$$

Following [12], we allow facility placement only on the set of nodes $N$. Any potential location which is not a node can be made to be a node and the coverage of a new arc that is incident to such a node can be proportionally adjusted. For example, if $w_{k l}=10$ and $d_{k l}=50$ and point $m$ at a distance of 5 from node $k$ on $\operatorname{arc}(k, l)$ is a potential location, for new $\operatorname{arc}(k, m), d_{k m}=5, w_{k m}=\left(\frac{5}{50}\right)(10)=1$ and for new arc $(m, l), d_{m l}=45, w_{m l}=\frac{45}{50}(10)=9$. Therefore we can change $G$ to $N$ in (1).

\subsection{The formulation of Church and Meadows [12]}

The formulation is based on the Network Intersect Point Set (NIPS), which is also the dominating set of points in $G$ for both the maximal node cover and arc cover problems. To define NIPS, Church and Meadows first define NIP as any point that is $R$ units of distance away from any node $i \in N$. Then, NIPS is the set of all NIPs and the nodes. Next, they call any segment between two adjacent points of NIPS (not including the two end points), a segment of equal coverage (SEC).

Notice that all points in a given SEC arc are covered by exactly the same nodes. Suppose there are $K$ SECs on the network.

Let $N\left(\mathrm{SEC}_{k}\right)$ be the set of all nodes that cover any point lying within $\mathrm{SEC}_{k}$ for $k=1, \ldots, K$.

Let

$$
\begin{aligned}
& y_{j}= \begin{cases}1 & \text { if a facility is located at node } j, \\
0 & \text { otherwise }\end{cases} \\
& z_{k}= \begin{cases}1 & \text { if } \mathrm{SEC}_{k} \text { is covered, } \\
0 & \text { otherwise }\end{cases}
\end{aligned}
$$

Let $A(k)$ be the arc that contains $\mathrm{SEC}_{k}$ and $w_{k}$ its weight. Let $v_{k}=w_{k} l\left(\mathrm{SEC}_{k}\right) / d_{A(k)}$ where $l\left(\mathrm{SEC}_{k}\right)$ and $d_{A(k)}$ are, respectively, the lengths of $\mathrm{SEC}_{k}$ and $\operatorname{arc} A(k)$. 


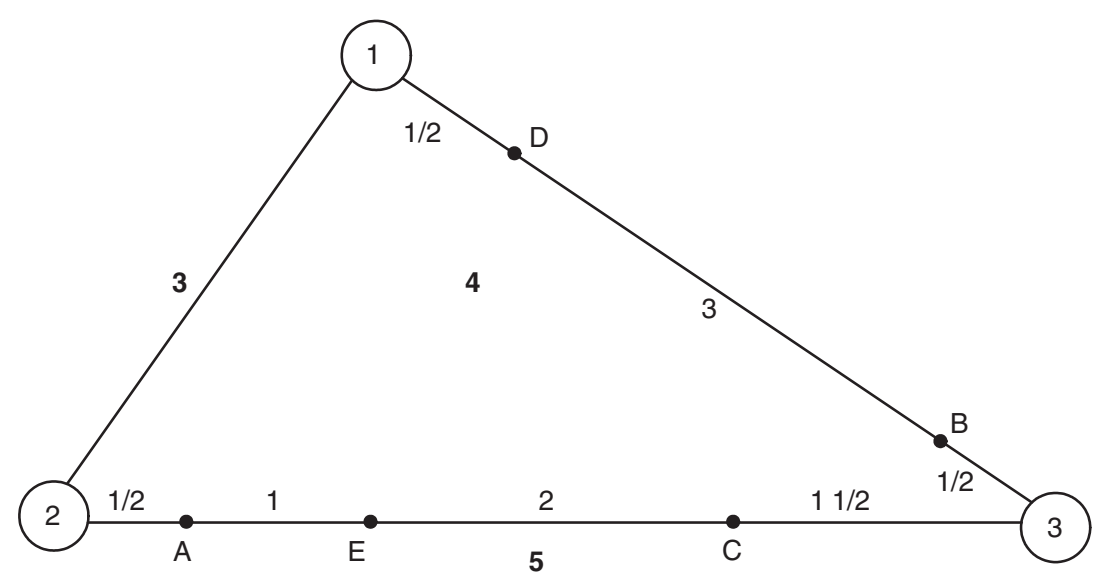

Fig. 1. A 3 node network.

The problem is

$\left(\mathrm{P}_{1}\right)$

$$
\begin{array}{ll}
\max & \sum_{k=1}^{K} v_{k} z_{k} \\
& \sum_{\substack{j \in N\left(\mathrm{SEC}_{k}\right)\\
}} y_{j} \geqslant z_{k}, \quad k=1, \ldots, K, \\
& \sum_{j=1}^{n} y_{j}=p, \\
& x_{j}, z_{k}=0,1 .
\end{array}
$$

Constraints (2) ensure that if $\mathrm{SEC}_{k}$ is covered only if there is a facility located at atleast one of the nodes that covers $\mathrm{SEC}_{k}$. In constraint (3) we limit the number of facilities to $p$. Church and Meadows use a commercial solver to solve this problem.

Example 1. Consider the three-node network, where $R=3 \frac{1}{2}$ and $p=2$ where the length of links is indicated in bold and length of segments in regular font (e.g. $l(1,2)=3$ and $\left.l_{2 A}=.5\right)$. Suppose all arc weights are equal to 1 (Fig. 1).

There are 8 SECs: 12, 2A, AE, EC, C3, 1D, DB, B3 with, respectively, corresponding variables $z_{1}, z_{2}, z_{3}, z_{4}, z_{5}, z_{6}, z_{7}, z_{8}$.

$\mathrm{NIPS}=\{1,2,3, A, B, C, D, E\}$. 
The problem is

$$
\begin{aligned}
\max & \left\{z_{1}+\frac{1}{10} z_{2}+\frac{1}{5} z_{3}+\frac{2}{5} z_{4}+\frac{3}{10} z_{5}+\frac{1}{8} z_{6}+\frac{3}{4} z_{7}+\frac{1}{8} z_{8}\right\} \\
\text { s.t. } & \\
& y_{1}+y_{2} \geqslant z_{1}, \\
& y_{1}+y_{2} \geqslant z_{2}, \\
& y_{2} \geqslant z_{3} \\
& y_{2}+y_{3} \geqslant z_{4} \\
& y_{3} \geqslant z_{5} \\
& y_{1}+y_{2} \geqslant z_{6}, \\
& y_{1}+y_{3} \geqslant z_{7}, \\
& y_{3} \geqslant z_{8}, \\
& y_{1}+y_{2}+y_{3}=2, \\
& y_{1}, y_{2}, y_{3}, z_{1}, z_{2}, \ldots, z_{8}=0,1 .
\end{aligned}
$$

In this problem there are 11 binary variables and nine linear constraints.

\subsection{The alternative formulation}

In addition to the binary variables $y_{j}$ defined earlier, we define $Z_{k l}$ as the fraction of arc $(k, l)$ covered. We also define parameters $a_{j k l}, j \in N,(k, l) \in A$, as the fraction of arc $(k, l)$ covered by a facility located at node $j$ through node $k$.

$$
a_{j k l}= \begin{cases}1 & \text { if } d(j, k)+d_{k l} \leqslant R, \\ \frac{R-d(j, k)}{d_{k l}} & \text { if } d(j, k) \leqslant R<d(j, k)+d_{k l}, \\ 0 & \text { otherwise. }\end{cases}
$$

We denote the fraction of coverage provided by the facility at node $j$ via node $l$ as $a_{j l k}$, which can be computed by replacing $d(j, k)$ with $d(j, l)$ in (4). Notice that the total coverage of arc $(k, l)$ by facility $j$ cannot exceed $100 \%$, and hence, to avoid double counting $Z_{k l}$ is equal to $\min \left\{a_{k l j}+a_{j l k}, 1\right\}$.

We can now formulate problem $\left(\mathrm{P}_{2}\right)$.

$\left(\mathrm{P}_{2}\right)$

$$
\begin{array}{ll}
\max & \sum_{(k, l) \in A} w_{k l} Z_{k l} \\
\text { s.t. } & \\
& \sum_{j \in N} y_{j}=p, \\
& \max _{j \in N}\left\{y_{j} a_{j k l}\right\}+\max _{j \in N}\left\{y_{j} a_{j l k}\right\} \geqslant Z_{k l} \quad(k, l) \in A, \\
& 0 \leqslant Z_{k l} \leqslant 1 \quad(k, l) \in A, \\
& y_{j}=0,1, \quad j \in N .
\end{array}
$$


Table 1

$a_{j k l}$ and $a_{j l k}$ value for Example 1

\begin{tabular}{lllllll}
\hline Node & \multicolumn{5}{l}{ Arcs $(k, l)$ and $(l, k)$} & \\
\cline { 2 - 7 } & $(1,2)$ & $(2,1)$ & $(1,3)$ & $(3,1)$ & $(2,3)$ & $(3,2)$ \\
\hline 1 & 1 & $\frac{1}{6}$ & $\frac{7}{8}$ & 0 & $\frac{1}{10}$ & 0 \\
2 & $\frac{1}{6}$ & 1 & $\frac{1}{8}$ & 0 & $\frac{7}{10}$ & 0 \\
3 & 0 & 0 & 0 & $\frac{7}{8}$ & 0 & $\frac{7}{10}$ \\
\hline
\end{tabular}

Together with the objective function, constraints (5) ensure that $Z_{k l}$ represents the total coverage provided to arc $(k, l)$ through nodes $k$ and $l$. Note that $Z_{k l}=0$ unless there are facilities that cover arc $(k, l)$. Also, (5) and (6) eliminate double-counting of any coverage overlap among $k$ and $l$.

For Example 1, the parameters $a_{j k l}$ and $a_{j l k}$ are summarized in Table 1.

The problem now can be formulated as follows:

$$
\begin{aligned}
\max & Z_{12}+Z_{13}+Z_{23} \\
\text { s.t. } & \\
& y_{1}+y_{2}+y_{3}=2, \\
& \max \left\{y_{1}, \frac{1}{6} y_{2}\right\}+\max \left\{\frac{1}{6} y_{1}, y_{2}\right\} \geqslant Z_{12}, \\
& \max \left\{\frac{7}{8} y_{1}, \frac{1}{8} y_{2}\right\}+\frac{7}{8} y_{3} \geqslant Z_{13}, \\
& \max \left\{\frac{1}{10} y_{1}, \frac{7}{10} y_{2}\right\}+\frac{7}{10} y_{3} \geqslant Z_{23}, \\
& 0 \leqslant Z_{12}, Z_{13}, Z_{23} \leqslant 1, y_{1}, y_{2}, y_{3}=\{0,1\} .
\end{aligned}
$$

The problem has 3 binary variables, 3 continuous variables, 4 constraints and 3 upper bound constraints.

Problem $\left(\mathrm{P}_{2}\right)$ is a mixed integer non-linear program, which we can solve using Branch and Bound. To obtain an upper bound, we solve the (LP) relaxation of a new problem called $\left(\mathrm{P}_{2}^{\prime}\right)$.

$\left(\mathrm{P}_{2}^{\prime}\right)$

$$
\begin{array}{ll}
\max & \sum_{(k, l) \in A} w_{k l} Z_{k l} \\
\text { s.t. } & \\
& \sum_{j \in N} y_{j}=p, \\
& \sum_{j \in N} y_{j} \min \left\{a_{j k l}+a_{j l k}, 1\right\} \geqslant Z_{k l} \quad(k, l) \in A, \\
& 0 \leqslant Z_{k l} \leqslant 1 \quad(k, l) \in A, \\
& y_{j}=0,1, \quad j \in N .
\end{array}
$$


Problems $\left(\mathrm{P}_{2}^{\prime}\right)$ and $\left(\mathrm{P}_{2}\right)$ are identical except for constraints (5) and (7). It is easy to verify that any solution that is feasible in $\left(\mathrm{P}_{2}\right)$ is also feasible in $\left(\mathrm{P}_{2}^{\prime}\right)$ and therefore $\left(\mathrm{P}_{2}^{\prime}\right)$ and certainly the $(\mathrm{LP})$ relaxation of $\left(\mathrm{P}_{2}^{\prime}\right)$ provides an upper bound for $\left(\mathrm{P}_{2}\right)$. Constraint $(7)$, in fact, overestimates $Z_{k l}$ by double-counting the coverage provided by different facilities. To illustrate, take two facilities $i, j \in N$ and $\operatorname{arc}(k, l) \in A$, where $d_{k l}=10, d(i, k)=7$ and $d(j, k)=5$. When coverage distance $R=8, a_{i k l}=0.1$ and $a_{j k l}=0.3$. In this example, the actual coverage of arc $(k, l)$ through $k$ is 0.3 , whereas adding up the coverage provided by nodes $i$ and $j$ as in (7) estimates the coverage as 0.4 .

Notice that if the optimal solution of the LP relaxation of $\left(\mathrm{P}_{2}^{\prime}\right)$ contains only integer values of the $y_{j}$ variables, this constitutes a feasible solution to $\left(\mathrm{P}_{2}\right)$ that may serve as a lower bound. In this case, we can calculate $w(S)$ using (1), where $S$ is the set of the $p y_{j}$ variables equal to 1 , and

$$
Z_{k l}(S)=\min \left\{\max _{j \in S}\left\{a_{j k l}\right\}+\max _{j \in S}\left\{a_{j l k}\right\}, 1\right\} .
$$

\subsection{Discussion}

It is easy to verify that Problem $\left(\mathrm{P}_{2}\right)$ has considerably less decision variables and constraints than $\left(\mathrm{P}_{1}\right)$. Although the non-linear formulation is much more compact, $\left(\mathrm{P}_{1}\right)$ is amenable to solution by a commercial solver. Our computational experience showed that solving $\left(\mathrm{P}_{1}\right)$ using CPLEX is more efficient than solving $\left(\mathrm{P}_{2}\right)$ using our Branch and Bound procedure (where CPLEX is used for solving the LP relaxation of $\left(\mathrm{P}_{2}^{\prime}\right)$ at each node of the tree). For example, for a randomly generated set of 30 problems, each with 150 nodes and 300 arcs, $\left(\mathrm{P}_{2}\right)$ required an average of $1.9 \mathrm{~h}$ CPU time, whereas an average of $35 \mathrm{~s}$ was sufficient for $\left(\mathrm{P}_{1}\right)$-excluding the pre-processing time for generating NIPS and SECs. However, the computational effort required for solving $\left(\mathrm{P}_{1}\right)$ increases drastically with problem size as our computational experiments reported in the next section demonstrate. CPLEX was unable to identify the optimal solution within $24 \mathrm{~h}$ for 22 of the 60 random instances of the 500-node problems we tackled. This calls for the development of a heuristic procedure for solving large-scale maximal arc-covering problems. As we will show later, a heuristic (e.g., a greedy heuristic) based on $\left(\mathrm{P}_{2}\right)$ is more efficient than its counterpart based on $\left(\mathrm{P}_{1}\right)$, since the latter involves the calculation of NIPS and all SECs in the pre-processing stage. Another advantage of $\left(\mathrm{P}_{2}\right)$ is that it is a direct formulation of the problem and hence easier to understand than $\left(\mathrm{P}_{1}\right)$. Although $\left(\mathrm{P}_{1}\right)$ is in the form of a maximal node-covering formulation, its decision variables are based on NIPS and SECs that are potentially less communicable to a decision maker.

The greedy heuristic described below is identical for Problems $\left(\mathrm{P}_{1}\right)$ and $\left(\mathrm{P}_{2}\right)$, except that for $\left(\mathrm{P}_{1}\right)$, it is required in Step 1 also to find NIPS and $\operatorname{SEC}_{k} \quad k=1, \ldots, K$ and in Step $2 W_{i}(S \cup\{i\})=\sum_{k=1}^{K} v_{k} g_{k}$ where $g_{k}=1$ if $(S \cup\{i\}) \cap N\left(\mathrm{SEC}_{k}\right) \neq \varnothing$ and $g_{k}=0$ otherwise. It is important to point out that both heuristics find the same solution to the maximal arc-covering problem.

\section{The Greedy Heuristic for $\mathbf{P}_{2}$}

Step 1: Set $S=\emptyset, j=0, N^{\prime}=N$

Step 2: $j=j+1$

For $i \in N^{\prime}$ Do

End for

$$
W_{i}(S \cup\{i\})=\sum_{(k, l) \in A} w_{k l} Z_{k l}(S \cup\{i\})(\operatorname{using}(8))
$$

$$
i^{*}=\arg \max _{i \in N^{\prime}} W_{i}(S \cup\{i\}), W^{*}=W_{i}\left(S \cup\left\{i^{*}\right\}\right), N^{\prime}=N^{\prime}-\left\{i^{*}\right\}, S=S \cup\left\{i^{*}\right\}
$$


Step 3: If $j=p$ or $N^{\prime}=\varnothing$ stop, $S$ is the "optimal" set of locations and $W^{*}$ is the optimal objective function. Otherwise go to Step 2.

For Example 1:

In Step 1: $S=\emptyset, j=0, N^{\prime}=\{1,2,3\}$.

In Step $2, j=1, W_{1}(\{1\})=\min \left\{1+\frac{1}{6}, 1\right\}+\min \left\{\frac{7}{8}+0,1\right\}+\min \left\{\frac{1}{10}+0,1\right\}=1.975$,

$W_{2}(\{2\})=\min \left\{\frac{1}{6}+1,0\right\}+\min \left\{\frac{1}{8}+0,1\right\}+\min \left\{\frac{7}{10}+0,1\right\}=1.825$,

$W_{3}(\{3\})=\min \{0+0,1\}+\min \left\{0+\frac{7}{8}, 1\right\}+\min \left\{\frac{7}{10}+0,1\right\}=.975$.

Therefore $i^{*}=1, W^{*}=1.975$ and $S=\{1\}$. Since $j<2$ in Step 3,j=2, it is easy to verify that $W_{2}(\{1,2\})=2.575$ and $W_{3}(\{1,3\})=2.8$ and therefore $i^{*}=\{3\}, S=\{1,3\}$ and $W^{*}=2.8$. Since $j=2$, $S=\{1,3\}$ is the greedy solution with the objective function value of 2.8 . We note that the optimal solution is $\{2,3\}$ with $W^{*}=3$.

\section{Computational experiments}

We tested the Church and Meadows formulation as well as the greedy heuristic based on our formulation on 60 randomly generated problem instances. The objective of the computational experiments was twofold: (i) to demonstrate the need for the development of a heuristic, and (ii) to investigate the quality of the solutions provided by the proposed heuristic in tackling large-scale problems. Naturally, these experiments also helped us to understand how problem parameters impact the required computational effort. The computations were carried out on a SUN HPC4500 workstation. The heuristic is implemented in C whereas CPLEX 7.1 is used for solving $\left(\mathrm{P}_{1}\right)$.

We randomly generated 10 distinct 500-node networks where the number of arcs is derived from a uniform distribution between 1000 and 3000. In order to ensure connectivity, we first randomly generated a tree with 500 nodes and then randomly added the remaining arcs to the network. The length of each arc $d_{i j}$ is derived from a uniform distribution in the interval $[1,10]$, and $w_{i j}=1$ for all arcs. $^{2}$ We solved six instances of the problem for each network by varying the number of response facilities to be located and the coverage distance i.e., $p$ and $R$. The $p$ values we used are 5 and 10 . We made sure that the networks do satisfy the triangle inequality. The $R$ values are $\left\lceil k\left(L_{1}+L_{2}\right)\right\rceil$ for $k=\frac{1}{6}, \frac{1}{3}$, and $\frac{1}{2}$, where $\lceil$.$\rceil denotes$ upper ceiling. $L_{1}$ and $L_{2}$ are the minimum and maximum path lengths in the node-to-node shortest path matrix. ${ }^{3}$

Table 2 reports on our experience with $\left(\mathrm{P}_{1}\right)$ and shows that the computational requirements of Church and Meadows formulation can be excessive for large-scale problems. To expose the non-monotonic computational requirements of $\left(\mathrm{P}_{1}\right)$, the 500-node networks are sorted in ascending order with respect to the number of arcs in the table. For the six instances associated with each network, the table depicts the (average, minimum and maximum) pre-processing times and total CPU times (i.e., pre-process plus CPLEX). We terminated CPLEX in the event that an optimal solution cannot be identified within $24 \mathrm{~h}$. In such cases, which occurred at least once for each network except for Network \#1 with the least arc density, we used $24 \mathrm{~h}$ in calculating the average total CPU time. The last column of Table 2 depicts the number of instances that was solved to optimality within $24 \mathrm{~h}$ for each 500 -node network we tackled.

\footnotetext{
${ }^{2}$ Variability in the benefit of covering each arc is incorporated in the next section.

${ }^{3}$ Each response team will presumably use the shortest path to reach the accident site from its facility.
} 
Table 2

Computational results with $\left(\mathrm{P}_{1}\right)$

\begin{tabular}{|c|c|c|c|c|c|c|c|c|}
\hline \multirow[t]{2}{*}{ Network \# } & \multirow[t]{2}{*}{ No. of arcs } & \multicolumn{3}{|l|}{ Pre-process time } & \multicolumn{3}{|c|}{ Total CPU time } & \multirow[t]{2}{*}{ No. of opt. (Out of 6} \\
\hline & & Average (min) & Min. (s) & Max. & Average & Min. (s) & Max. (h) & \\
\hline 1 & 1073 & 44 & 135 & $1.5 \mathrm{~h}$ & $56 \min$ & 304.6 & 1.5 & 6 \\
\hline 9 & 1235 & 40 & 95.8 & $1.6 \mathrm{~h}$ & $8.9 \mathrm{~h}$ & 899 & $>24$ & 4 \\
\hline 4 & 1347 & 40 & 91.5 & $1.7 \mathrm{~h}$ & $8.7 \mathrm{~h}$ & 106.1 & $>24$ & 4 \\
\hline 10 & 1418 & 36 & 64.6 & $1.5 \mathrm{~h}$ & $8.4 \mathrm{~h}$ & 83.6 & $>24$ & 4 \\
\hline 5 & 2130 & 51 & 72.08 & $3.7 \mathrm{~h}$ & $14 \mathrm{~h}$ & 82.92 & $>24$ & 3 \\
\hline 2 & 2150 & 18 & 76 & $36 \min$ & $12.5 \mathrm{~h}$ & 83 & $>24$ & 3 \\
\hline 6 & 2221 & 46 & 162 & $1.7 \mathrm{~h}$ & $12.5 \mathrm{~h}$ & 304 & $>24$ & 3 \\
\hline 3 & 2313 & 21 & 90 & $39 \min$ & $12.2 \mathrm{~h}$ & 100 & $>24$ & 3 \\
\hline 8 & 2496 & 51 & 127 & $1.9 \mathrm{~h}$ & $8.3 \mathrm{~h}$ & 135 & $>24$ & 4 \\
\hline 7 & 2587 & 52 & 158 & $1.7 \mathrm{~h}$ & $6.9 \mathrm{~h}$ & 176 & $>24$ & 4 \\
\hline
\end{tabular}

Table 3

Detailed results for network \#5

\begin{tabular}{|c|c|c|c|c|c|c|c|}
\hline \multirow[t]{2}{*}{$R$} & \multirow[t]{2}{*}{$P$} & \multicolumn{4}{|c|}{ Optimum (s) } & \multicolumn{2}{|l|}{ Heuristic } \\
\hline & & Prep. & Opt. & Total & Opt. & CPU (s) & Gap (\%) \\
\hline 4 & 5 & 72.08 & 10.84 & 82.92 & $\mathrm{Y}$ & 2.14 & 0.001 \\
\hline 4 & 10 & 72.08 & 20.31 & 92.39 & Y & 6.78 & 2.33 \\
\hline 9 & 5 & 1609.2 & $>24 h$ & $>24 \mathrm{~h}$ & $\mathrm{~N}$ & 2.28 & -33 \\
\hline 9 & 10 & 1608.5 & $>24 h$ & $>24 \mathrm{~h}$ & $\mathrm{~N}$ & 7 & -13 \\
\hline 13 & 5 & 5369.7 & $>24 h$ & $>24 h$ & $\mathrm{~N}$ & 2.29 & 0.06 \\
\hline 13 & 10 & 9535.8 & 38765 & 48300.7 & $\mathrm{Y}$ & 7.1 & 0.03 \\
\hline
\end{tabular}

During our experiments with $\left(\mathrm{P}_{1}\right)$, we observed that pre-processing can take a considerable time, which increases with $R$. The average pre-processing time of the shortest coverage distances for each of the 10 networks is $1.8 \mathrm{~min}$. This increases to $27.4 \mathrm{~min}$ and $90.3 \mathrm{~min}$ as $R$ is increased to $\left(L_{1}+L_{2}\right) / 3$ and $\left(L_{1}+L_{2}\right) / 2$, respectively. Observe that in some instances the pre-processing time takes hours. As expected, the computational requirements increase with the number of facilities $p$ as well.

Now, we focus on Network \#5 with 2130 arcs, which proved particularly difficult for the Church and Meadows formulation. The computational details of the six instances for this network are depicted in Table 3. In addition to the results of $\left(\mathrm{P}_{1}\right)$, the table also includes our findings via the proposed heuristic based on $\left(\mathrm{P}_{2}\right)$. The percent gap, depicted in the last column, is calculated by dividing the difference between the best solution from $\left(\mathrm{P}_{1}\right)$ and the heuristic solution with the former value. Note that the best solution from $\left(\mathrm{P}_{1}\right)$ was optimal only for three instances of this network. More importantly, for $R=9$ and $p=5$ CPLEX cannot improve the solution after $24 \mathrm{~h}$ and terminates with an optimality gap of $38 \%$. This solution has a coverage value of 1301 , whereas the heuristic solves the problem in $2.3 \mathrm{~s}$ with a solution that gives 1731 coverage (i.e., $81 \%$ of the 2130 arcs are covered in the heuristic solution). The negative gap in Table 3 for this instance indicates that the heuristic indeed finds a 33\% better solution than the 
Table 4

Computational results with the greedy heuristic based on $\left(\mathrm{P}_{2}\right)$

\begin{tabular}{|c|c|c|c|c|c|}
\hline \multirow[t]{2}{*}{ Network \# } & \multirow[t]{2}{*}{ No. of arcs } & \multirow[t]{2}{*}{ CPU average (s) } & \multicolumn{3}{|l|}{ Gap (\%) } \\
\hline & & & Average & Best & Worst \\
\hline 1 & 1073 & 2.2 & 0.23 & 0 & 0.7 \\
\hline 9 & 1235 & 2.6 & 1 & 0 & 3.2 \\
\hline 4 & 1347 & 4.3 & 0.118 & -0.13 & 8 \\
\hline 10 & 1418 & 3.0 & 1.1 & 0 & 3.5 \\
\hline 5 & 2130 & 4.6 & -7.26 & -33 & 2.3 \\
\hline 2 & 2150 & 4.6 & -0.57 & -4 & 0.6 \\
\hline 6 & 2221 & 4.8 & -5.2 & -24 & 0.5 \\
\hline 3 & 2313 & 5.0 & -0.52 & -3 & 1.2 \\
\hline 8 & 2496 & 5.4 & -1.98 & -14.6 & 4.7 \\
\hline 7 & 2587 & 5.6 & -0.238 & -38 & 1.7 \\
\hline
\end{tabular}

Church and Meadows formulation. Interestingly, we were able to obtain a lower bound from the Branch and Bound procedure on $\left(\mathrm{P}_{2}\right)$ in 4 min that is 1718 - which is clearly better than the best solution provided by $\left(\mathrm{P}_{1}\right)$ for this instance.

Table 4 presents a summary of our experiments with the heuristic procedure on the same 60 instances. Note that, on the average, all of the 10 networks were solved within $6 \mathrm{~s}$. As per the worst case performance of the heuristic, the most challenging 500-node instance took less than nine seconds to solve. More importantly, the average gaps are negative for those networks with more than 2000 arcs i.e., the heuristic provided a better solution than that of CPLEX in $24 \mathrm{~h}$. Although the gap can be as high as $38 \%$ in favor of the heuristic (see Network \#7 in the table), it is never worse than $8 \%$ in favor of $\left(\mathrm{P}_{1}\right)$.

We note that we have not warm-started CPLEX with the result of the heuristic. We conjecture that it will allow CPLEX to find the optimal solution in less time.

Finally, it is obvious that our greedy heuristic dominates a greedy heuristic based on the Church and Meadows formulation that will incur the pre-processing times in Table 3 required to calculate the NIPS and SECs. We also believe that combining our heuristic with another procedure like an exchange routine can improve the heuristic further without significantly increasing the CPU time.

\section{The Quebec-Ontario problem}

In this section,we study the emergency response infrastructure for gasoline incidents on the highway networks of Quebec and Ontario in Canada. Our aim is to provide an assessment of the coverage provided by the current system and to demonstrate the improvement that can be achieved via the proposed methodology. Verter and Kara [13] showed that Geographical Information Systems (GIS) are essential means for large-scale risk assessment endeavors. Thus, we derived the model parameters from a GIS-based representation of the two provinces. We used ArcView 3.1 GIS software to superimpose three layers of digital maps: the population zones, the road network and the origin-destination points for each shipment. This enabled us to estimate the route choices of hazmat carriers and the resulting level of population 
exposure around each highway link. The coverage provided by each alternative response team station was also determined via the GIS-based representation.

The data set for this application is compiled from a number of sources. Statistics Canada keeps a record of each hazmat shipment including its origin, destination, type of material, number of truck-loads and monetary value. These records indicate that 102,420 truck-loads of gasoline were carried between 251 origin-destination pairs in 1998. Transport Canada [2] requires evacuation of the people residing within $800 \mathrm{~m}$ of a gasoline spill. We obtained population data from the 1996 census, which contains 1149 sub-divisions in Quebec and 543 sub-divisions in Ontario. Most of these zones have very low population density. Thus, our model includes only those census sub-divisions with population density higher than 40 people $/ \mathrm{km}^{2}$. This amounts to 108 zones in Quebec with a total population of 3.8 million and 72 zones in Ontario with a total population of 7.6 million. Our highway network model is based on the ArcView 3.1 map and it has 205 arcs and 176 nodes. An arc corresponds to an urban road if it lies within a population center and it is considered a rural road otherwise. We assume that response teams travel $50 \mathrm{~km} / \mathrm{h}$ on urban roads and $90 \mathrm{~km} / \mathrm{h}$ on rural roads. These average travel speeds translate coverage distances to response times.

To derive problem parameters, we assume that hazmat carriers use the shortest path for each shipment. This allows us to estimate the number of gasoline trucks on each highway link. Through GIS, we also determine the number of people living within $800 \mathrm{~m}$ of each link. The multiplication of these two numbers for a highway link gives the total population exposure (in truck-people), which we use as a proxy of the benefit of providing full coverage to the link i.e., $w_{i j}$. Interestingly, only 95 of the 205 arcs have $w_{i j}>0$ in this instance, and $w_{i j}$ has a large range of values. We avoid potential stability problems during the solution process by normalizing arc weights in the model i.e., we divide each $w_{i j}$ by $\max _{i j} w_{i j}$. The highway link with maximum population exposure is in Toronto. The total benefit of covering all the 95 arcs with $w_{i j}>0$ is 5.0513, which we use as a benchmark.

There are 23 gasoline shipment origins in Quebec and Ontario with a wide range of activity levels. Four of these origins account for $78 \%$ of the 102,420 shipments, ${ }^{4}$ whereas three origins ship less than 50 gasoline trucks annually. The major shipment origins in the two provinces are depicted in Fig. 2. As mentioned in Section 1, hazmat shippers in Canada are responsible from emergencies during transport. Most major shippers, such as Shell, PetroCanada and Ultramar, employ their own specialized response team, whereas small-scale shippers often outsource their ERAP to a third party. Thus, we represent the existing emergency response network via a response team station at each shipment origin, except for the three smallest sites. Our calculations indicate that the current 20-team system requires more than $1.5 \mathrm{~h}$ to provide full coverage. More specifically, the total coverage within $90 \mathrm{~min}$ is 4.9507 , which amounts to $98 \%$ of the benchmark.

The current specialized response team locations certainly provide convenience in stationing the teams and their equipment. Intuitively, however, the shipment origins are not necessarily the best sites for maximizing coverage to possible road incidents. We analyzed the extent of improvement that can be achieved via optimizing the emergency network design. To facilitate the analysis, we solved the problem for response times of 30,60 and $90 \mathrm{~min}$. We solved the problem using the Branch and Bound algorithm from $\left(\mathrm{P}_{2}\right)$. Table 5 provides a comparison of the coverage provided by the current and proposed response networks. Note that the proposed 20 -team network covers $97 \%$ of the highway network within $30 \mathrm{~min}$,

\footnotetext{
${ }^{4}$ Toronto 30,440 trucks, La Cote de Beupre 28,561 trucks, Laval(North of Montreal) 10,758 trucks and Brant County 10,577 trucks.
} 


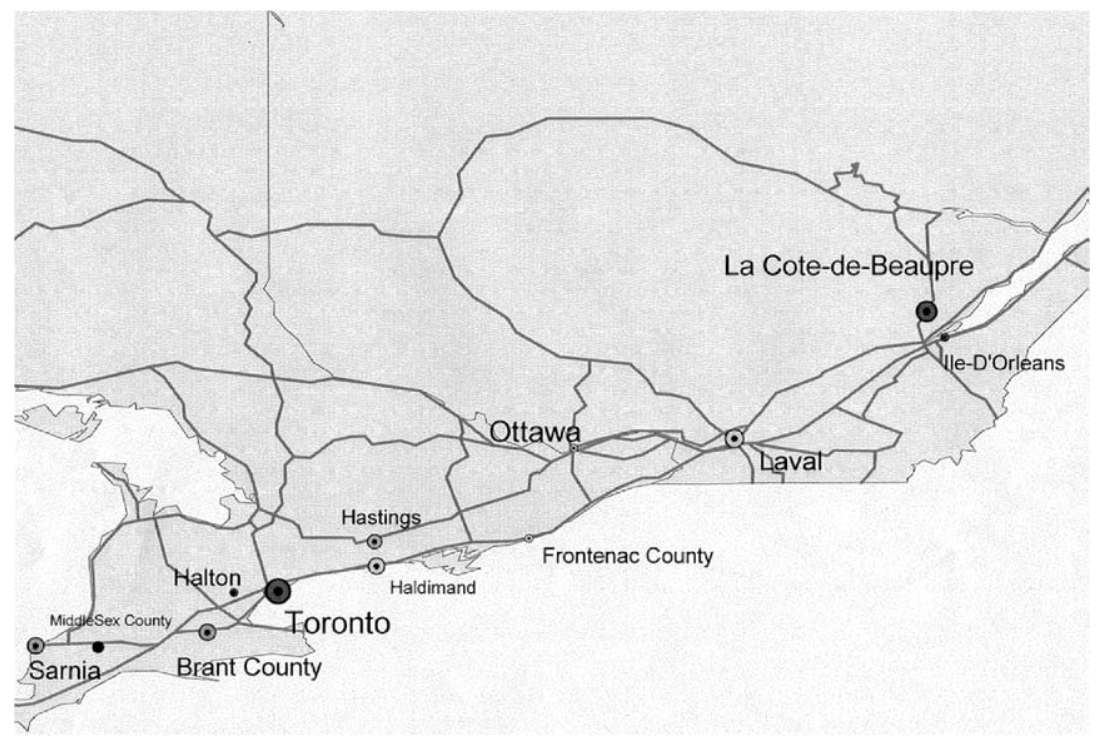

Fig. 2. Origins of major gasoline shipments (size of circular points is proportional to the annual amount of shipment).

Table 5

Coverage percentages for the Quebec-Ontario problem

\begin{tabular}{llll}
\hline Response time (min) & Current 20-team & Proposed 20-team & Proposed 14-team \\
\hline 30 & 59.8 & 96.7 & 96.7 \\
60 & 92.2 & 98.1 & 97.5 \\
90 & 98.0 & 99.9 & 99.9 \\
\hline
\end{tabular}

whereas the current system needs about 90 min to provide the same coverage. In other words, it is possible to achieve a remarkable $67 \%$ reduction in the threshold time that define coverage for gasoline incidents by optimizing the location of the 20 response teams.

Re-location of response team stations requires a capital outlay, which may be excessive for small-scale shippers. Therefore, in re-designing the emergency response network, we focus on the shipments that involve more than 1000 truck-loads of gasoline annually. There are 14 such shipment origins, and hence Table 5 also depicts the coverage statistics for the proposed 14-team network. Interestingly, the percent coverage remains almost at the same level despite the $30 \%$ reduction in the number of response teams. The optimal solution provides $97.9 \%$ coverage. More importantly, only two of the 14 teams are located at their current site in the optimal solution.

Provincial governments of Quebec and Ontario have jurisdiction over highway shipments of dangerous goods. The above analysis, however, adopts a holistic approach in modeling the highway networks of the two provinces as a single entity. There are obvious challenges associated with the implementation of such a multi-province solution. To determine the benefits of the holistic approach, we also optimized each province's response network in isolation. Currently, there are 5 response stations in Quebec and 
15 stations in Ontario. For a response time of $30 \mathrm{~min}$, the maximum coverage of the 5-team network in Quebec is 1.7 and that of the 15-team network in Ontario is 3.19. Thus, the total coverage provided in the two provinces is 4.89 , which is equal to the coverage provided by the holistic approach (i.e., $96.7 \%$ of the benchmark as depicted in Table 5). The additional coverage provided by streamlining the provincial networks is also marginal for response times of 60 and $90 \mathrm{~min}$. Consequently, the time and effort required for coordinating the emergency response network re-design processes in the two provinces would be hard to justify.

\section{Concluding comments}

Time is of the essence in responding to hazmat incidents. Quick arrival of a specialized team at the incident site can reduce the undesirable impact considerably. Response time depends on the distance between the specialized team station and the incident site. Therefore, the locations of response team stations constitute a major factor in the design of an emergency response network for dangerous goods incidents in a region. In this paper, we present the maximal arc-covering location model as a means to optimize the structure of an emergency response network. We discussed two formulations to solve the maximal arc-covering location problem, a known one and a new one. An application of the proposed methodology in Quebec and Ontario pointed out the possibility of a significant improvement in response capability via relocation of the existing stations. Naturally, the implementation phase requires an agreement between the provincial governments and the hazmat shippers with regard to the allotment of relocation costs.

The likelihood that a response team will be busy at the time of an incident has attracted considerable attention in the maximal covering literature since the seminal work of Daskin [14]. Given the very low probabilities associated with hazmat incidents, however, the occurrence of multiple accidents within the response area of a specialized team is highly unlikely. Therefore, in the context of hazmat transportation, we can safely assume that the response teams will be available at all times.

The notion of gradual covering decay, recently proposed by Berman et al. [15], provides a promising avenue for future research. In this paper, we followed the traditional approach in considering a potential accident site "uncovered," if a response team cannot reach this point within the threshold time. In responding to a hazmat incident, however, a specialized team can still mitigate some of the impact despite arriving after the threshold time. The incorporation of gradual covering decay requires the use of a dynamic transport risk model that accounts for the time distribution of undesirable consequences.

Recently, Kara and Verter [16] developed a model to determine the road network that can be used for hazmat shipments so as to minimize the total transport risk in a region. The integration of their hazmat network design model with the model proposed in this paper is non-trivial from a solution methodology perspective. Nonetheless, it would be an important step toward the development of a comprehensive transport risk mitigation policy.

\section{Acknowledgements}

This research was partially funded by two discovery grants from the Natural Sciences and Engineering Research Council (NSERC) of Canada. The insights provided by Robert Lapalme, Coordinator of Major Risk Management at Quebec Department of Public Security, are greatly appreciated. The authors are 
grateful to three anonymous referees and the guest editor whose comments and suggestions were helpful in improving the paper.

\section{References}

[1] US Department of Transportation. Departmentwide program evaluation of the hazardous materials transportation programs. 2000. http://hazmat.dot.gov/hmpe_report.pdf

[2] Transport Canada. Emergency response guidebook 2000. 2000. Download from http://www.tc.gc.ca/canutec/en/ guide/guide.htm

[3] Lapalme R. Personal Communication. 2003.

[4] US National Transportation Safety Board. Safety recommendation. R-99-7 through-10. 1999. Download from http:// hazmat.dot.gov/ntsb_safety.htm

[5] US National Transportation Safety Board. Safety recommendation. R-01-22. 2001. Download from http://hazmat.dot.gov/ ntsb_safety.htm

[6] Minot Daily News. Hoeven updates state on minot train derailment. January 18. 2002.

[7] Erkut E, Verter V. Modeling of transport risk for hazardous materials. Operations Research 1998;46:625-42.

[8] Verter V, Lapierre S. Location of preventive health care facilities. Annals of Operations Research 2002;110:123-32.

[9] Church RL, ReVelle C. The maximal covering location problem. Papers of Regional Science Association 1974;32: 101-18.

[10] Marianov V, Serra D. Location problems in the public sector. In: Drezner Z, Hamacher HW, editors. Facility location: applications and theory. Berlin: Springer; 2002. [Chapter 4].

[11] ReVelle C, Toregas, Falkson. Applications of the location set-covering problem. Geographical analysis 1976;7:65-75.

[12] Church RL, Meadows ME. Location modeling utilizing maximum service distance criteria. Geographical Analysis 1979;11:358-73.

[13] Verter V, Kara B. A GIS-based framework for hazardous materials transport risk assessment. Risk Analysis 2001;21: 1109-20.

[14] Daskin MS. A maximum expected covering location model: formulation, properties and heuristic solution. Transportation Science 1983;17:48-70.

[15] Berman O, Krass D, Drenzer Z. The gradual covering decay location problem on a network. European Journal of Operational Research 2003;151:474-80.

[16] Kara B, Verter V. Designing a road network for hazardous materials transportation. Transportation Science 2004;38: 188-96. 